\title{
Table-top two-color soft X-ray laser by means of Ni-like plasmas
}

\author{
Leili Masoudnia, ${ }^{1}$ Mabel Ruiz-Lopez, ${ }^{1}$ and Davide Bleiner ${ }^{1,2, a)}$ \\ ${ }^{1}$ Swiss Federal Laboratories for Materials Science and Technology (Empa), Überlandstrasse 129, \\ CH-8600 Dübendorf, Switzerland \\ ${ }^{2}$ University of Zürich, Rämistrasse 71, CH-8006 Zürich, Switzerland
}

(Received 25 October 2015; accepted 4 April 2016; published online 19 April 2016)

Laser-produced Ni-like plasmas are known as active media for extreme ultraviolet lasing, with the flexibility to two-color lasing. Two-color laser generation is very complex at accelerator facilities. In this work, plasma lasing at the $3 \mathrm{~d}^{9} 4 \mathrm{~d}^{1}(\mathrm{~J}=0) \rightarrow 3 \mathrm{~d}^{9} 4 \mathrm{p}^{1}(\mathrm{~J}=1)$ (collisional-pumping process) and the $3 \mathrm{~d}^{9} 4 \mathrm{f}^{1}(\mathrm{~J}=1) \rightarrow 3 \mathrm{~d}^{9} 4 \mathrm{~d}^{1}(\mathrm{~J}=1)$ (photo-pumping process) transitions is studied experimentally and computationally. Several key characteristics of collisional- and photo-pumping laser, such as divergence, pointing stability, and intensity have been investigated. The measurements showed different pulse characteristics for the two lasing processes affected by plasma inhomogeneity in temperature and density. Analytical expressions of these characteristics for both collisional- and photopumping are derived. It is found that the plasma that maximizes the photo-pumping lasing is $20 \%$ hotter and $70 \%$ denser than the plasma that optimizes the collisional-pumping lasing. The gain of collisional pumping is $\approx 4$ times higher than the gain for the photo-pumping. The gain lifetime is a factor of $\approx 5.2$ larger for the monopole-pumping. Similarly, the gain thickness is a factor of $\approx 1.8$ larger. It is also found that the gain build-up time for collisional- and photo-pumping is $0.7 \mathrm{ps}$ and $0.9 \mathrm{ps}$, respectively, whereas the build-up length-scale is $11.5 \mu \mathrm{m}$ and $6.3 \mu \mathrm{m}$, respectively. Published by AIP Publishing. [http://dx.doi.org/10.1063/1.4946883]

\section{INTRODUCTION}

The X-ray pulses with bimodal spectra ("two-color") are very insightful pump-probes to investigate the structure of matter, the dynamics of physical processes, and chemical reactions. ${ }^{1,2}$ Theoretically, the timescales of rotation, vibration, dissociation of molecules, atomic excitation, atomic ionization, etc., are known. ${ }^{3,4}$ Ideally, one would measure experimentally such timescales by time-resolved means using dual X-ray pulses with specific time delays and spectral separation. Furthermore, near-edge X-ray absorption spectroscopy to simultaneously probe the structure and surface chemistry is another application for two-color probes. ${ }^{5}$ Besides, the soft X-ray high energy resolution off-resonance spectroscopy (HEROS) technique by means of two-color can be a powerful method to study the local electronic and geometric structures of matter. ${ }^{6-8}$

In 2013, SLAC researchers demonstrated for the first time the pairs of X-ray laser pulses ${ }^{1,9}$ through self-amplified spontaneous emission (SASE) by means of X-ray Free-Electron Lasers, with wavelengths at $\approx 0.81 \mathrm{~nm}(E \approx 1530.7 \mathrm{eV})$ and $\approx 0.83 \mathrm{~nm}(E \approx 1493.8 \mathrm{eV})$, i.e., a spectral separation as small as $0.2 \AA$. The SPring- 8 Angstrom Compact free electron LAser facility can currently produce pairs of $\mathrm{X}$-ray laser pulses at $\approx 0.09 \mathrm{~nm}\left(E \approx 13^{\prime} 776 \mathrm{eV}\right)$ and $\approx 0.12 \mathrm{~nm}\left(E \approx 10^{\prime} 332 \mathrm{eV}\right){ }^{2}$ The two radiation pulses were delayed from coincidence to a maximum delay as long as $40 \mathrm{fs}{ }^{1}$

Two pulses with different photon energies ("two-color pulses") have been generated in an accelerator, ${ }^{1,2,9,10}$ using three different schemes: (i) double split undulator mode (Fig. 1(a)), (ii) gain-modulated undulator mode (Fig. 1(b)), and

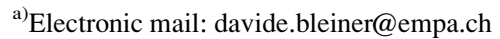

(iii) double-bunch undulator mode (Fig. 1(c)). The double split undulator mode (see Fig. 1(a)) is divided into two parts, with a magnetic delay (magnetic chicane) placed in the middle. ${ }^{1,2}$ In this scheme, the wavelengths of the two X-ray pulses are given by the expression: $\lambda_{1,2}=\lambda_{w} \frac{1+K_{1,2}^{2}}{2 \gamma^{2}}$, where $\lambda_{w}, K$, and $\gamma$ are, respectively, the undulator wavelength, undulator parameter, and electron Lorentz factor. The "magnetic delay" between the two undulators allows a temporal compression of the electron bunch, i.e., the rotation of electron bunch in the energy-time plane. Since both X-ray pulses are emitted by the same electron bunch, the first of the two pulses cannot be amplified to saturation. Indeed, the energy-spread induced by the X-ray free-electron lasers (XFELs) process would prevent lasing on the second color.

The gain-modulated undulator mode (see Fig. 1(b)) is composed of the sections of alternating magnetic field strength. ${ }^{10}$ Here, the wavelengths of two radiated X-rays are equal to $\lambda_{1,2}=\lambda_{w} \frac{1+K_{1,2}^{2}}{2 \gamma^{2}}$. Since the two colors are emitted by the same electron bunch, the same limitation as above applies. In addition, the spectrum of a gain-modulated XFEL can exhibit more than two peaks, due to complex spectral features arising from the interference of the radiation emitted in two consecutive resonant undulators. ${ }^{1,10}$

The double-bunch undulator mode (see Fig. 1(c)) is performed by using two electron bunches with different energies and overlapped in time, so that two wavelengths appear at the exit of the undulator with a separation in the wavelength..$^{9,11,12}$ In this scheme, the wavelengths of two radiated $\mathrm{X}$-rays are equal to $\lambda_{1,2}=\lambda_{w} \frac{1+K^{2}}{2 \gamma_{1,2}^{2}}$. All these are possible at large scale facilities, with the limitation in spectral separation of the two colors. 


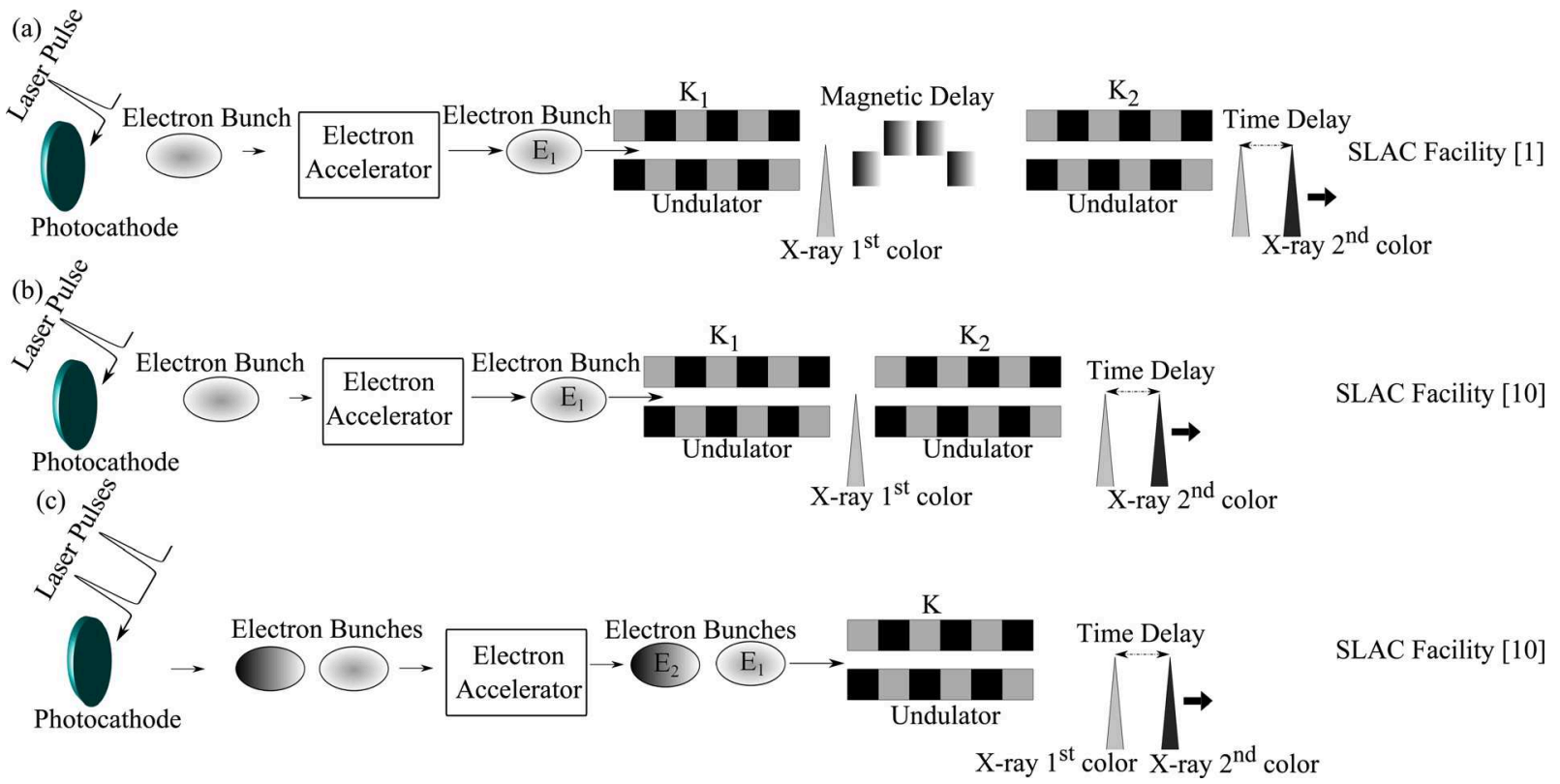

FIG. 1. Layout of the two-color options at accelerator facilities: ${ }^{1,10}$ (a) double split undulator mode, (b) gain-modulated undulator mode, and (c) double-bunch undulator mode.

X-ray free-electron laser (XFEL) facilities are currently available in a few sites, due to the remarkable cost of construction and operation. ${ }^{13}$ In this regard, the laboratory-scale two-color extreme ultraviolet (XUV) plasma-based laser can be a more widely accessible platform. ${ }^{14}$

It has been demonstrated at the LLNL that the laserproduced plasmas are able to produce two XUV coherent pulses by focusing an intense laser pump on a target. ${ }^{15}$ Two XUV lasers produced by a target have wavelengths separated by as much as $5.1 \mathrm{~nm}(\Delta E=10.5 \mathrm{eV})$. In the laser-produced plasma, lasing lines come from a given radiator (e.g., Mo) through two different pumping processes: (i) collisionalpumping (monopole: $\Delta J=0, J=0 \rightarrow 0$ ) and (ii) photopumping (dipole: $\Delta J=1, J=0 \rightarrow 1$ ) processes. The collisional laser line is pumped through electron collisions and is the result of the transition $3 \mathrm{~d}^{9} 4 \mathrm{~d}^{1}(\mathrm{~J}=0) \rightarrow 3 \mathrm{~d}^{9} 4 \mathrm{p}^{1}(\mathrm{~J}=1)$ for $\mathrm{Ni}$-like ions. On the other hand, the photo-pumped laser line is pumped by a strong radiation (e.g., $\Delta E \simeq 353 \mathrm{eV}$ $\equiv 3.51 \mathrm{~nm}$ for Ni-like Mo) producing a laser line (XUV), which is the result of the transition $3 \mathrm{~d}^{9} 4 \mathrm{f}^{1}(\mathrm{~J}=1) \rightarrow$ $3 \mathrm{~d}^{9} 4 \mathrm{~d}^{1}(\mathrm{~J}=1)$ for Ni-like ions.

Fig. 2(a) summarizes two-color XUV wavelengths generated by electron collisional-pumping and photo-pumping in Ni-like ions with different plasma lasing schemes. In Ref. 15 , a $1 \mathrm{~J}$ pulse with the pulse duration of 600 ps illuminates the $\mathrm{Nb}$ target $\left(\mathrm{Nb}^{+13}\right)$ followed $700 \mathrm{ps}$ later by a $5 \mathrm{~J}$ and $1 \mathrm{ps}$ pulse. In Ref. 16, lasing in $\mathrm{Zr}^{+12}$ is produced by the combination of a long pulse ( $800 \mathrm{ps})$ and a short pulse (1 ps) with the delay between the two pulses set at $1.6 \mathrm{~ns}$, and the maximum energy in the long and short pulses each being 5 J. In Ref. 17, the scheme for lasing is irradiating the Mo target $\left(\mathrm{Mo}^{+14}\right)$ with a $4 \mathrm{~J}, 1.5 \mathrm{~ns}$ pulse followed by a $3 \mathrm{~J}, 1 \mathrm{ps}$ pulse.

The photo-pumped four-level system (dipole transition) and collisionally excited three-level system (monopole transition) for Ni-like ions are shown in Fig. 2(b). Indeed, Nilike ions with electron configuration of $[\mathrm{Ar}] 3 \mathrm{~d}^{10}$ are excited through monopole or dipole transition (see Fig. 2(b)).
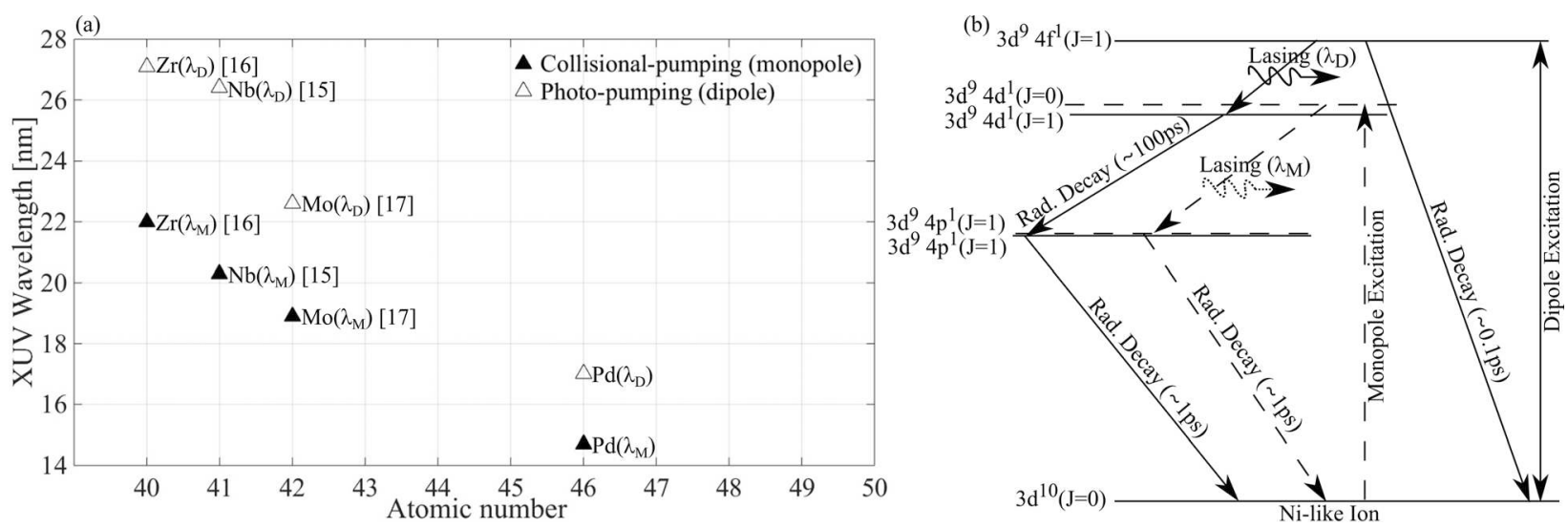

FIG. 2. (a) Plasma laser wavelength versus atomic number that exhibit dual wavelength emission due to photo-pumping $\left(\lambda_{D}\right)$ and electron collisional-pumping $\left(\lambda_{M}\right)$ in Ni-like ions. ${ }^{15-17}$ It shows for the Ni-like ion: $\lambda_{M}<\lambda_{D}$. (b) The four-level system (solid-line) due to the dipole and three-level system (dashed-line) due to the monopole transition for Ni-like atom. Time scales of radiative decays (Rad-decay) are the order of magnitude. 
Here, the aim is to demonstrate the key characteristics of two-color XUV lasers generated in the own lab within a micro-plasma active medium of Ni-like $\mathrm{Mo}\left(\mathrm{Mo}^{+14}\right)$ and Nilike $\mathrm{Pd}\left(\mathrm{Pd}^{+18}\right)$. Thus, the characteristics of two-color XUV lasers, such as divergence and pointing stability, are obtained experimentally. Then, computational results to investigate plasma hydro-characteristics at the lasing region for the two XUV laser lines are presented. The time difference that maximizes the gain for two-color emission in the plasma medium is obtained. Here, it is mainly interesting to investigate the hydrodynamics and atomic physics descriptions and difference between the characteristics of plasmas producing collisionally pumped and photo-pumped lines.

This work is organized as follows: In Sec. II, a description of experimental set-up and performing the experiment are presented. Furthermore, a description of modeling codes is given. In Sec. III, the approach used in the theoretical calculation is explained. In Secs. IV A 1 and IV A 2, the characterization of XUV lasers due to photo-pumping and collisional-pumping for Ni-like Mo and Pd is shown, respectively. In Sec. IV B 1, the computational analysis and interpretation for collisional- and photo-pumping are presented. In Sec. IV B 2, an explanation on the temporal and spatial distribution of the gain is presented. A comparison between computational and experimental results is represented. In Sec. IV C, the XUV wavelengths due to photo-pumping for some Ni-like ions are investigated.

\section{MATERIAL AND METHODS}

\section{A. Experiment}

\section{Pump laser and target}

In the in-house set-up, a planar target is used, i.e., the plasma is created by a $\lambda=1054 \mathrm{~nm}$ Nd-glass chirped pulse amplification (CPA) laser system ${ }^{18}$ with a pulse duration of 1.2 ps (FWHM) and Gaussian pulse shape. The beams including two pre-pulses and a main-pulse are irradiated at an incident angle of $40^{\circ}$ with respect to the target normal, which leads to a line focus. ${ }^{19,20}$ Two $0.5 \%$ and $16 \%$ prepulses (which are produced by inserting two beam splitters) irradiated the target $4.8 \mathrm{~ns}$ and $0.5 \mathrm{~ns}$ before the arrival of the main pulse for Mo. They arrived $5 \mathrm{~ns}$ and $0.2 \mathrm{~ns}$ before the arrival of the main pulse in the case of the Pd target. The pre-pulses were collinear with the main pulse. The total optimized-energy of the pulses in the experiment fluctuates in the range of $2.5 \pm 0.1 \mathrm{~J}$ for Mo and $2.6 \pm 0.1 \mathrm{~J}$ for Pd on a line focus of $12 \mathrm{~mm} \times 65 \mu \mathrm{m}$, see Fig. 3 .

\section{Flat field-spectrometer}

A flat field grazing incidence spectrometer is used to acquire spectra of the plasma-driven XUV laser, see Fig. 3. The XUV laser emission was measured on-axis using a 1200 grooves/mm, aberration-corrected, flat-field Hitachi grating. ${ }^{21}$ The spectrum was recorded on a phosphor screen (P43) imaged onto a cooled CCD camera having a pixel size of $23 \mu \mathrm{m}$. The slit-less spectrometer resolves the image with a spectral resolution of $\pm 0.2 \mathrm{~nm}$. But an "entrance pinhole" has been implemented in order to narrow the opening of the spectrometer and improve the resolution up to $\pm 0.01 \mathrm{~nm}$, see Fig. 3.

\section{B. Computation}

\section{Boundary conditions for computation}

For the calculation, only a Mo planar target (see Fig. 3) was chosen along with an electronic flux-limit of 0.05 and a multi-group radiation transport of 50 groups which are logarithmically interpolated the photon energy domain in the range of $1 \mathrm{eV}-10 \mathrm{keV}$. In addition, it is considered that the peak of first pre-pulse arrives at the target at $2.4 \mathrm{ps}$. The prepulses and main-pulse have a Gaussian pulse shape. Furthermore, the total energy of the pulse train is $2.5 \mathrm{~J}$. The time precision for the computation is considered $0.5 \%$ of ps.

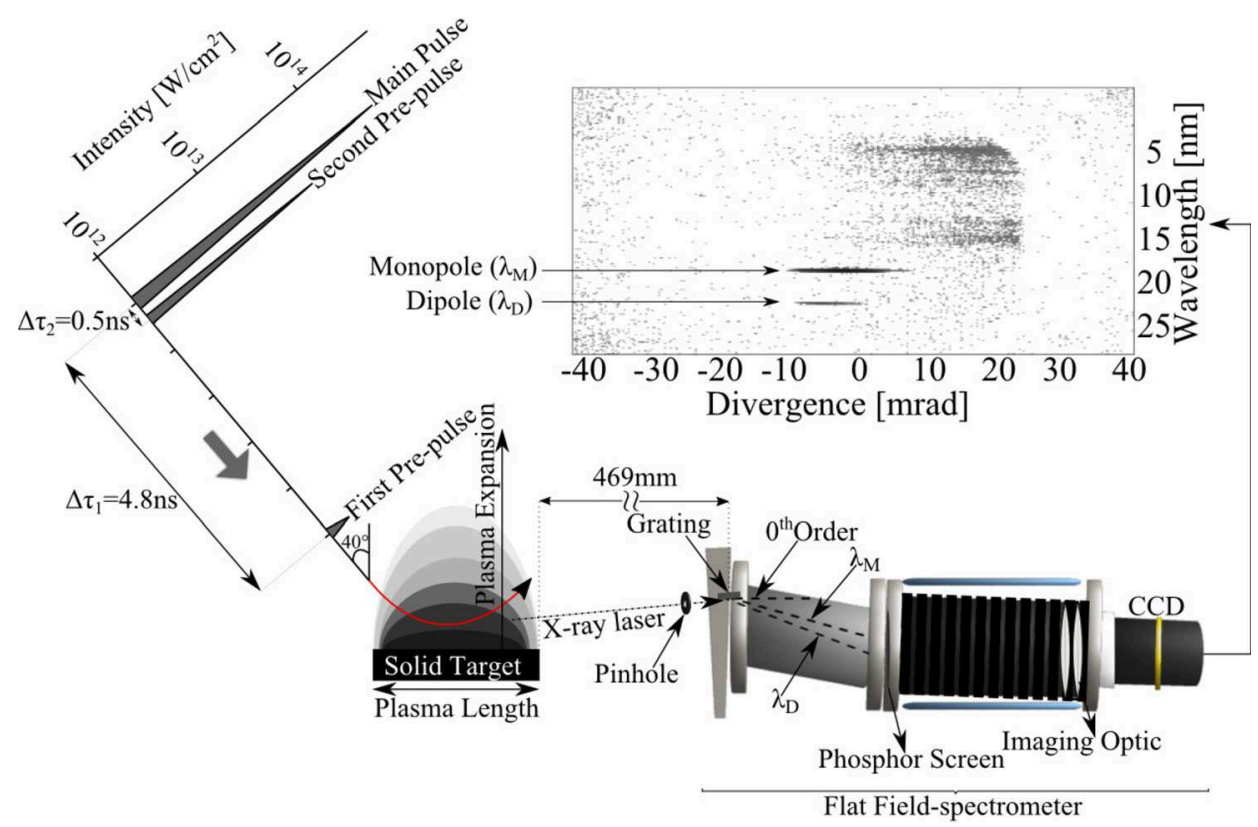

FIG. 3. The scheme of two collinear pre-pulses and the main pulse (TGRIP scheme) that irradiate the planar target at a fixed angle. All pre-pulses and the main pulse are fed to the target (Mo and Pd) along the same beam-line. $\Delta \tau_{1}$ and $\Delta \tau_{2}$ stand for the delay time between the first pre-pulse and secondpre pulse with respect to the mainpulse. A flat field-spectrometer is used to acquire spectra of X-ray laser produced by plasma. 


\section{Computational tools}

a. Hydrodynamic code. "HYADES",22,23 is a onedimensional, three-geometry (planar, cylindrical or spherical), three-fluid (electrons, ions, and radiation) hydrodynamics computation code. The conservation equations for mass, momentum, and energy are solved in a Lagrangian system. The three fluids are treated individually in a fluid approximation, each having its own temperature. Each fluid is assumed to be in local thermodynamic equilibrium, which is to say, that the electrons and the ions are described well in the classical limit by Maxwell-Boltzmann statistics, and the radiation field is Planckian. Electron degeneracy effects, important at lowtemperature, high-density plasmas, are taken into account. The equation of state (EOS) and related thermodynamic coefficients are obtained from external tables that have been compiled using experimental data and theoretical models. The energy transport by free-electrons and ions is modeled in the flux-limited diffusion approximation (the default value for both electrons and ions is 0.4). Radiation is transported according to the photon energy, i.e., extreme ultraviolet and soft X-rays have very short mean free paths, while the more energetic photons' harder X-rays will penetrate deeply into the material. The absorption and emission coefficients are determined self-consistently from the atomic physics model of choice or may be supplied by the user in tabular form. The absorption of laser light by a hot plasma is dominated by inverse Bremsstrahlung at the intensity of interest to this work. The good agreement of HYADES computation with experimental values was verified. ${ }^{23,24}$ The HYADES code is since then extensively used in the community. ${ }^{23-29}$

b. Atomic physics code. The flexible atomic code $(\mathrm{FAC})^{30}$ is used to calculate the required atomic data, such as collisional excitation coefficients and spontaneous emission coefficients. It is a configuration interaction program for calculating atomic collisional and radiative processes, including (i) energy levels, (ii) radiative transitions and the inverse process of (iii) photo-excitation, (iv) collisional excitation and (v) ionization by electron impact and its inverse process, (vi) collisional de-excitation and (vii) three-body recombination, (viii) radiative recombination and its inverse process, (ix) photo-ionization, (x) auto-ionization and its inverse process, and (xi) dielectronic capture. These are indeed the required coefficients that we need to solve the nonlocal thermodynamic equilibrium (NLTE) system of rate equations. The FAC employs a fully relativistic approach based on the Dirac equation. $\mathrm{Gu}^{30}$ verified the good agreement of the FAC with experimental data. Since then, the FAC has been extensively used for calculating the atomic data in several publications. $^{28-34}$

\section{THEORY}

\section{A. Atomic model of the gain medium}

For the calculation of the XUV gain produced by collisional-pumping a three-level system is examined as shown in Fig. 4(a). For the calculation of the XUV gain produced
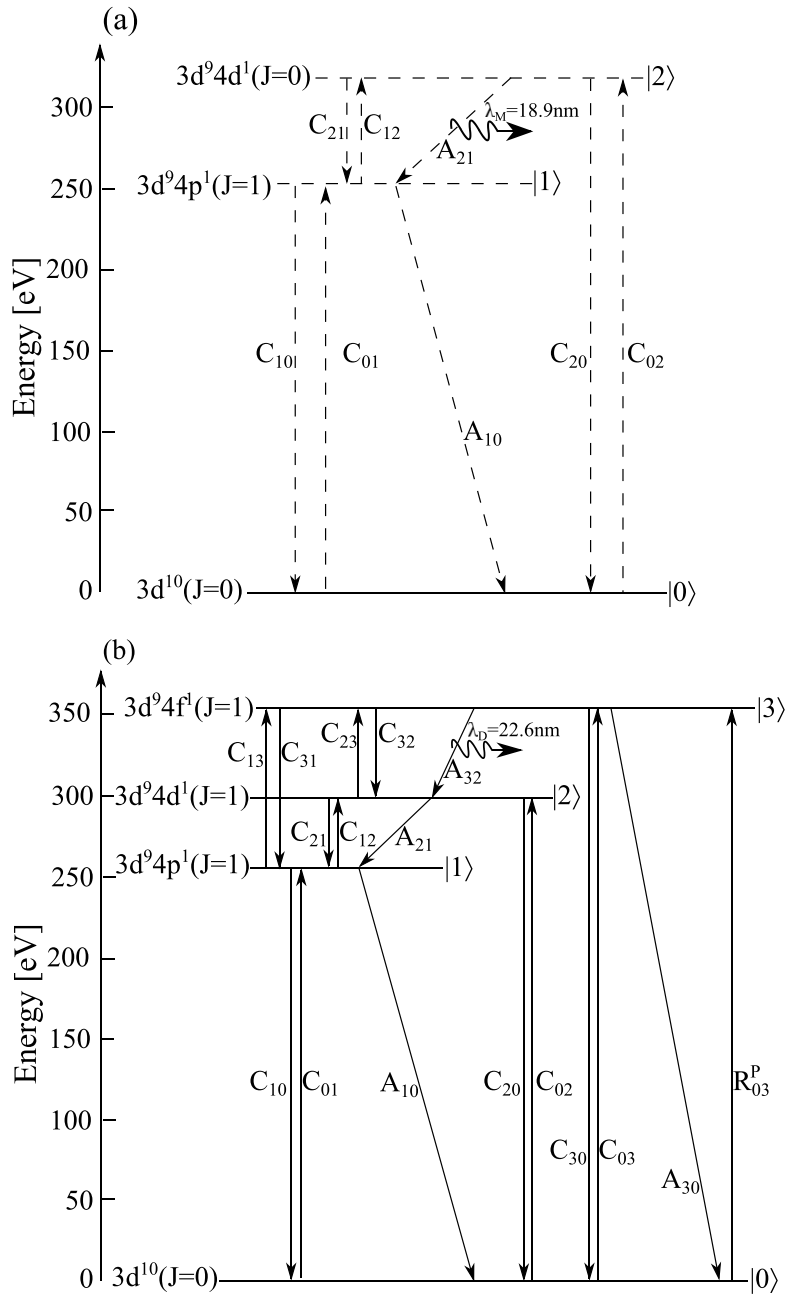

FIG. 4. (a) Grotrian scheme for monopole lasing $\left(\lambda_{M}\right)$ of the Ni-like Mo. (b) Grotrian scheme for dipole lasing $\left(\lambda_{D}\right)$ of the Ni-like Mo.

by photo-pumping a four-level system is examined as shown in Fig. 4(b).

\section{Calculation of gain due to the electron collisional-pumping (monopole mode)}

Fig. 4(a) shows a three-level system composed of the upper $|2\rangle$, lower $|1\rangle$, and the ground $|0\rangle$ state pumped by a transient collisional-pumping (collisional excitation) scheme. Due to the selection rule, the transition from the lower level $(\mathrm{J}=0)$ to the upper level $(\mathrm{J}=0)$ is only achieved by the monopole collisional excitation, and the photopumping (dipole excitation) is not allowed here (see Fig. 4(a)). Considering the steady state in a three-level model, rate equations are solved taking into account all the populating, depopulating, and radiative processes ${ }^{28,29}$

$$
\frac{d n_{i}}{d t}=n_{e}\left(\sum_{i<j} n_{i} C_{i j}^{e}+\sum_{i>j} n_{i} C_{i j}^{d}\right)+\sum_{i>j} n_{i} A_{i j} .
$$

Calculation of $n_{i}$, the population of the corresponding level $|i\rangle$, and calculation of gain due to the collisional-pumping (monopole) have been extensively explained in the previous publications. $^{28,29}$ 


\section{Calculation of gain due to the photo-pumping (dipole mode)}

Fig. 4(b) shows the lasing due to the photo-pumping for a four-level system. It schematically shows that the energy levels are composed of the upper $|3\rangle$ and lower $|2\rangle$ lasing states and intermediate level $|1\rangle$ and the ground $|0\rangle$ state. The transition from lower level $(\mathrm{J}=0)$ to upper level $(\mathrm{J}=1)$ is achieved via both the dipole collisional excitation $\left(C_{03}\right)$ and photo-excitation $\left(R_{03}^{P}\right)$, as shown in Fig. 4(b).

The upper level $|3\rangle$ is populated by both electron collisional-pumping and photo-pumping. The level populations of Ni-like ions are computed by solving the steadystate rate equations of a four-level laser model

$$
\frac{d n_{i}}{d t}=n_{e}\left(\sum_{i<j} n_{i} C_{i j}^{e}+\sum_{i>j} n_{i} C_{i j}^{d}\right)+\sum_{i>j} n_{i} A_{i j}+\sum_{i<j} n_{i} R_{i j}^{P} .
$$

Here, $C_{i j}^{e}$ and $C_{i j}^{d}$ are, respectively, collisional excitation and de-excitation rates in $\mathrm{cm}^{3} / \mathrm{s} . A_{i j}$ and $R_{i j}^{P}$ are the spontaneous emission and photo-pumping rates, respectively. In a four level system, it is found

$$
\begin{aligned}
& n_{1}=\Omega, \\
& n_{2}=\Omega \Theta+\phi, \\
& n_{3}=\frac{\Omega(\gamma+\Theta \beta)+\phi \beta+\Psi}{\alpha},
\end{aligned}
$$

where $n_{i}$ is the population of the corresponding level $|i\rangle$, and: $\Psi=n_{0}\left(n_{e} C_{03}+R_{03}^{P}\right), \quad \eta=n_{0} n_{e} C_{02}, \alpha=A_{32}+A_{30}+n_{e}\left(C_{32}\right.$ $\left.+C_{30}+C_{31}\right), \quad \gamma=n_{e} C_{13}, \quad \Lambda=C_{32} n_{e}+A_{32}, \zeta=n_{e}\left(C_{21}\right.$ $\left.+C_{23}+C_{20}\right)+A_{21}, \quad \Xi=C_{12} n_{e}, \quad \chi=n_{e} C_{21}+A_{21}, \quad \Upsilon=A_{10}$ $+n_{e}\left(C_{10}+C_{12}+C_{13}\right), \Delta=n_{0} n_{e} C_{01}, \beta=C_{23} n_{e}, \varrho=C_{31} n_{e}$, $\Theta=\frac{\gamma \Lambda+\Xi \alpha}{\zeta \alpha-\beta \Lambda}, \phi=\frac{\Psi \Lambda+\eta \alpha}{\zeta \alpha-\beta \Lambda}, \Omega=\frac{\phi(\beta \varrho+\chi \alpha)+\Psi \varrho+\Delta \alpha}{\gamma \alpha-\gamma \varrho-\Theta(\beta \varrho+\chi \alpha)}$.

The photo-pumping rate $\left(R_{03}^{P}\right)$ is determined as follows: $:^{35}$

$$
R_{03}^{P}\left[s^{-1}\right]=\frac{n_{p h}}{1+n_{p h}} \frac{\gamma_{3}}{\gamma_{0}} A_{30},
$$

where $\gamma_{i}, \gamma_{j}$ are the degeneracies of the $\mathrm{i}$-th, $\mathrm{j}$-th levels, and the degeneracy of each level is $2 \mathrm{~J}+1$ (statistical weight). $n_{p h}$ is the number of photons per mode, which is given as follows: ${ }^{36}$

$$
n_{p h}=\frac{1}{e^{h \nu / k T_{\text {rad }}-1}},
$$

where $k T_{r a d}$ is the radiation temperature of the pump-line. The gain given as the small signal gain coefficient describes the amplification in the system without considering its origin. ${ }^{37}$ The small signal gain is given by the expression below:

$$
g_{0}=n_{3} \sigma_{s t i m}-n_{2} \sigma_{a b} \approx n_{3} \sigma_{s t i m} F
$$

where $\sigma_{s t i m}, \sigma_{a b}$ are the cross section for stimulated emission and absorption, where the cross section for the stimulated emission is ${ }^{37}$

$$
\sigma_{\text {stim }}=1.3 \times 10^{-36} A_{32} \frac{\lambda^{3}}{\Delta \lambda / \lambda},
$$

where $\lambda$ is in Ångström units and $\Delta \lambda / \lambda$ is the relative spectral bandwidth. It is obtained through convolution of Doppler broadening $\left(\lambda_{D}\right)$ and collisional broadening $\left(\lambda_{L}\right){ }^{38}$ In a plasma, Doppler broadening due to the thermal motion of ions produces a Gaussian-shaped line function, where $\Delta \lambda_{D}=\frac{\sqrt{2 k T_{\text {ion }} / M}}{c} \lambda$. Here, $k T_{\text {ion }}$ is the ion temperature and $M$ is the ion mass.

The electron impact broadening produces a Lorentzianshaped line function, where $\Delta \lambda_{L} \propto n_{e} .{ }^{39}$ The inversion factor $(F)$ is the population inversion factor, defined as follows:

$$
F=1-\left(\frac{\gamma_{3}}{\gamma_{2}}\right) \frac{n_{2}}{n_{3}},
$$

where $\gamma_{i}$ and $\gamma_{j}$ are the degeneracies of the $\mathrm{i}$-th and $\mathrm{j}$-th levels. The degeneracy of each level is $2 \mathrm{~J}+1$ (statistical weight).

\section{B. Lasing process and saturation}

Assuming a line profile with a width of $\Delta \lambda$, the saturation intensity is ${ }^{40,41}$

$$
I_{s a t}=\frac{8 \pi h c^{2}}{\lambda^{4}} \frac{\Delta \lambda}{\lambda} .
$$

The upper limit for total X-ray intensity can be approximated by following equation: ${ }^{42}$

$$
I_{\text {tot }}=I_{\text {sat }} g_{0} L,
$$

where $g_{0} L$ is the small signal gain-length product obtained by considering

$$
g_{0} L=\int_{0}^{L} g_{0}(z) d z
$$

where $d z$ is along the target surface.

\section{Light propagation in the gain medium}

\section{XUV ray-tracing}

An important effect limiting the gain is refraction, which bends the pump away from the high gain zone. In this subsection, the ray trajectory is analyzed for $2 \mathrm{D}$ propagation. Considering the Maxwell's equations and the eikonal equation, ${ }^{43}$ namely,

$$
\frac{d}{d s}\left(n(\mathbf{r}, \omega) \frac{d \mathbf{r}}{d s}\right)=\nabla n(\mathbf{r}, \omega),
$$

where $d s$ is the element of distance along the ray, $\mathbf{r}$ is the position vector for a ray point, and $n$ is the refractive index of the medium

$$
n=\sqrt{1-\frac{n_{e}}{n_{c}}},
$$


where $n_{c}$ is the critical electron density at the frequency of the propagating ray

$$
n_{c}\left[\mathrm{~cm}^{-3}\right]=\frac{1.1 \times 10^{21}}{\lambda^{2}},
$$

where $\lambda$ is the wavelength in $\mu \mathrm{m}$.

The XUV refraction angle turns out to be given by the following expression: ${ }^{44,45}$

$$
\phi_{r}=\sqrt{\frac{n_{e 0}}{n_{c}}}
$$

where $n_{c}$ is the critical density, $n_{e 0}$ is the maximum electron density within the gain region, and $\phi_{r}$ is refraction angle from horizontal axis at which a ray exits the side of the plasma.

\section{Divergence and pointing-stability}

Pointing stability and divergence are properties used to characterize laser or laser-like radiation source such as a plasma based X-ray laser or an XFEL. For diagnosing the pointing stability and the divergence of the $\mathrm{X}$-ray pulses, a flat-field spectrometer is used, as shown in Fig. 3. The vertical axis on the CCD image is wavelength. The horizontal axis provides spatial resolution which can be converted into pulse divergence for any single shot. A series of shots may be used to obtain information on pointing stability as follows. The pointing stability is given by spatial fluctuation of the maximum peak of intensity (Gaussian-function) of the images obtained and calculated as the standard deviation $(1 \sigma$ precision) of the peak coordinates. A variation in the range of mrad is expected for table-top systems. ${ }^{46}$ Distance to the target and density of the plasma are related to the refraction angle of XUV lasers. The latter determines the coordinates of the maximum peak of intensity. The data are given in mrad in terms of the mean, the uncertainty, and the standard deviation of the experimental measurements. The divergence is introduced as the full width at $1 / \mathrm{e}^{2}$ of peak intensity.

\section{RESULTS AND DISCUSSION}

\section{A. Experimental XUV laser characterization}

\section{Molybdenum two-color laser}

Two-color XUV lasing at $\lambda_{M}=18.9 \mathrm{~nm}\left(E_{M}=65.6 \mathrm{eV}\right)$ and $\lambda_{D}=22.6 \mathrm{~nm}\left(E_{D}=54.9 \mathrm{eV}\right)$ generated by electron collisional-pumping and photo-pumping in Ni-like Mo were observed. The measured spectrum showed that the two wavelengths form Mo exhibit different pulse characteristics, such as pointing stability and divergence. These are affected by plasma inhomogeneity, e.g., temperature or density gradients. ${ }^{29}$

Fig. 5(a) shows the mean intensity for lasing lines observed in the experiment form the Mo laser. It shows that the peak intensity of XUV lasing at $\lambda_{M}=18.9 \mathrm{~nm}$ is a factor of $\approx 18$ higher than the peak intensity at $\lambda_{D}=22.6 \mathrm{~nm}$. Fig. 5 (b) shows the experimentally measured spectrum. Since there is free propagation of the XUV in the horizontal direction giving information about the XUV divergence, Fig. 5(c) shows that $10.2 \pm 1.0 \mathrm{mrad}$ and $8.3 \pm 1.4 \mathrm{mrad}$ are divergences determined for the full width of the profile at $1 / \mathrm{e}^{2}$ of the peak intensity for $\lambda_{M}=18.9 \mathrm{~nm}$ and $\lambda_{D}=22.6 \mathrm{~nm}$, respectively. Fig. 5(c) also shows that the mean peak intensity of each of the two wavelengths appears on the CCD-detector shifted by some $3.2 \mathrm{mrad}$. Table I summarizes the mean of
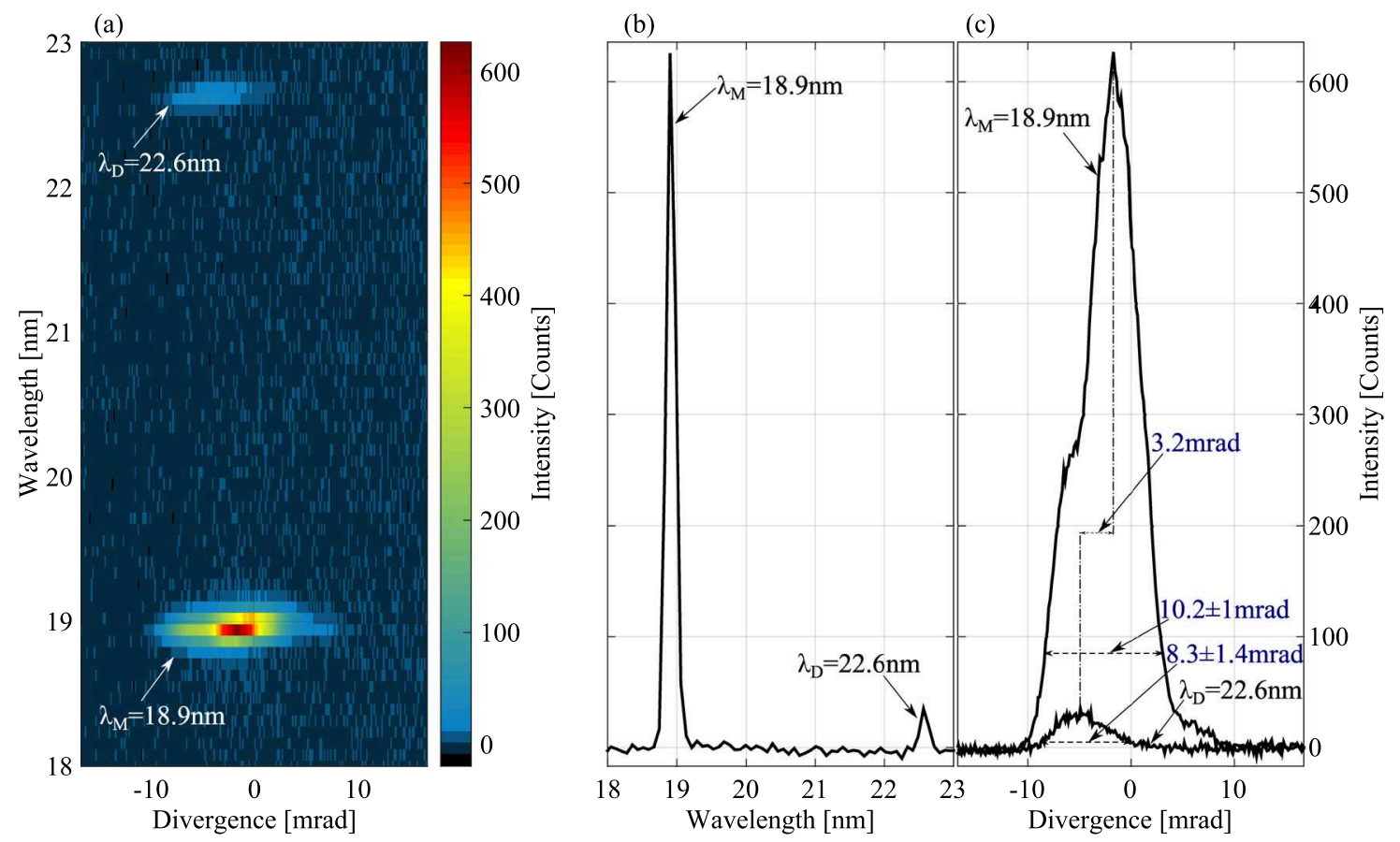

FIG. 5. (a) The experimentally measured mean intensity for lasing lines observed for Ni-like Mo at wavelengths $\lambda_{M}=18.9 \mathrm{~nm}$ and $\lambda_{D}=22.6 \mathrm{~nm}$. (b) Wavelength profile (vertical axis in (a)). (c) Divergence profile (horizontal axis in (a)). In subplots (a) and (c), the number 0 at the $x$-axis is the center of the image on the CCD-detector. 
TABLE I. Experimentally measured dual pulse characteristics (mean) of XUV laser lines observed in the experiment for Mo.

\begin{tabular}{lccc}
\hline \hline $\begin{array}{l}\text { Wavelength } \\
(\mathrm{nm})\end{array}$ & $\begin{array}{c}\text { Divergence } \\
(\text { mean) (mrad) }\end{array}$ & $\begin{array}{c}\text { Pointing } \\
\text { stability (mrad) }\end{array}$ & $\begin{array}{c}\text { Relative bandwidth } \\
(\Delta \lambda / \lambda)\end{array}$ \\
\hline$\lambda_{M}=18.9$ & $10.2 \pm 1.0$ & 1.4 & $9 \times 10^{-3}$ \\
$\lambda_{D}=22.6$ & $8.3 \pm 1.4$ & 1.4 & $6 \times 10^{-3}$ \\
\hline \hline
\end{tabular}

pointing stability, divergence, and intensity of two-color produced irradiating Mo target. The measured divergence at $\lambda_{M}=18.9 \mathrm{~nm}$ is $\approx 1.2$ times larger than the experimental divergence at $\lambda_{D}=22.6 \mathrm{~nm}$. The relative bandwidth $(\Delta \lambda / \lambda)$ of 18.9 is $50 \%$ larger than the relative bandwidth of $22.6 \mathrm{~nm}$. It is noteworthy to mention about the instrumental limitations concerning the real linewidth. In fact, based on the calculation $\Delta \lambda / \lambda \approx 8 \times 10^{-5}$, while the measured relative bandwidth is, due to the instrumental broadening as also discussed in Refs. 47-49, as high as shown in Table I.

\section{Palladium two-color laser}

Two-color XUV wavelengths at $\lambda_{M}=14.7 \mathrm{~nm}$ $\left(E_{M}=84.3 \mathrm{eV}\right)$ and $\lambda_{D}=17.3 \mathrm{~nm}\left(E_{D}=71.7 \mathrm{eV}\right)$ generated by electron collisional-pumping and photo-pumping in $\mathrm{Ni}$ like Pd were observed. The measured spectrum showed that two laser emission wavelengths from Pd exhibit different pulse characteristics.

Fig. 6(a) shows the mean intensity for lasing lines observed in the experiment with $\mathrm{Pd}$. It shows that the peak intensity of the XUV laser at $\lambda_{M}=14.7 \mathrm{~nm}$ is $\approx 10$ times higher than the peak intensity of the XUV laser at $\lambda_{D}=17.3 \mathrm{~nm}$. Fig. 6(b) shows the experimentally measured spectrum. In Fig. 6(c), $9.0 \pm 0.4$ and $6.8 \pm 1.4$ are divergences determined for the full width of the profile at $1 / \mathrm{e}^{2}$ of the peak intensities at wavelengths $\lambda_{M}=14.7 \mathrm{~nm}$ and $\lambda_{D}=17.3 \mathrm{~nm}$, respectively. Fig. 6(c) shows that the mean peak intensities of each of the two wavelengths appear on the CCD-detector shifted by some $1 \mathrm{mrad}$.

Table II summarizes the divergence of the XUV laser lines observed in the experiment with $\mathrm{Pd}$ for an optimizedtotal pulse energy $2.6 \pm 0.1 \mathrm{~J}$ (see Fig. 6). It shows that the experimentally measured divergence at wavelength $\lambda_{M}=14.7 \mathrm{~nm}$ is $\approx 1.3$ times larger than the experimental divergence at $\lambda_{D}=17.3 \mathrm{~nm}$. The relative bandwidth $(\Delta \lambda / \lambda)$ of 14.7 is $30 \%$ larger than the relative bandwidth of $17.3 \mathrm{~nm}$.

\section{B. Computational analysis and interpretation}

\section{Two-color XUV lasing process}

Fig. 7 shows the lifetime of each level due to radiative decay, where dashed lines represent a three-level system (collisional-pumping) and solid lines represent a four-level system (photo-pumping). For the Ni-like ions, a small population in the $4 \mathrm{f}$ state is expected because its fluorescence is fast $(\tau=0.1 \mathrm{ps})$. The $3 \mathrm{~d} \rightarrow 4 \mathrm{f}$ transition would be expected to be optically thick and so it creates a radiation field in the plasma that photon pumps electrons from the $3 \mathrm{~d}$ ground state $(|0\rangle)$ to the $4 \mathrm{f}$ excited state $(|3\rangle)$. This process ensures a sufficient population in the $4 \mathrm{f}$ state so that it can lase to the $4 \mathrm{~d}$ state. This in turn requires a high enough density so that collisions depopulate the $4 \mathrm{~d}$ lower laser level. The lifetime of ground level is estimated from the required ionization time of ground level $\left(\mathrm{Mo}^{+14}\right)$, where $t[s]=10^{11} / n_{e}\left[\mathrm{~cm}^{-3}\right] .50$

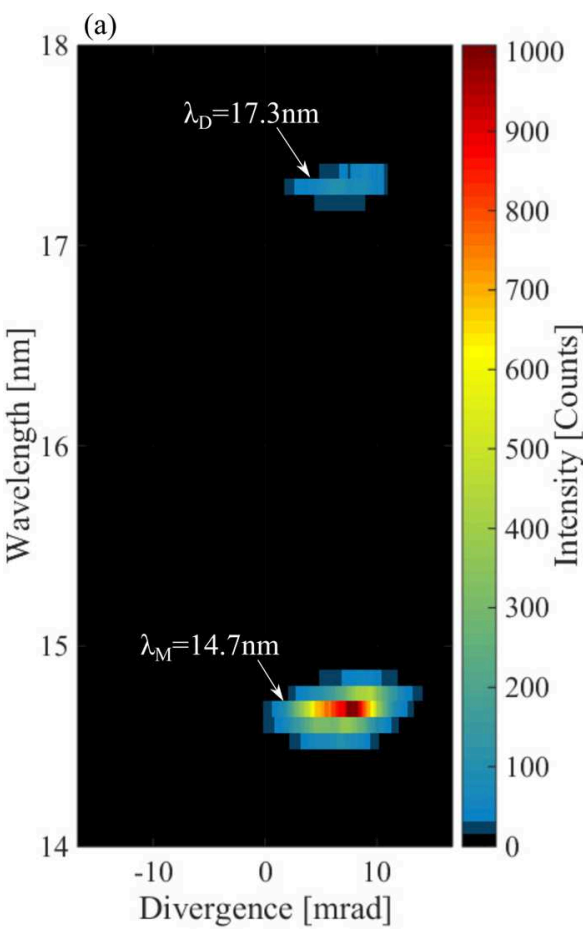

(b)

(c)

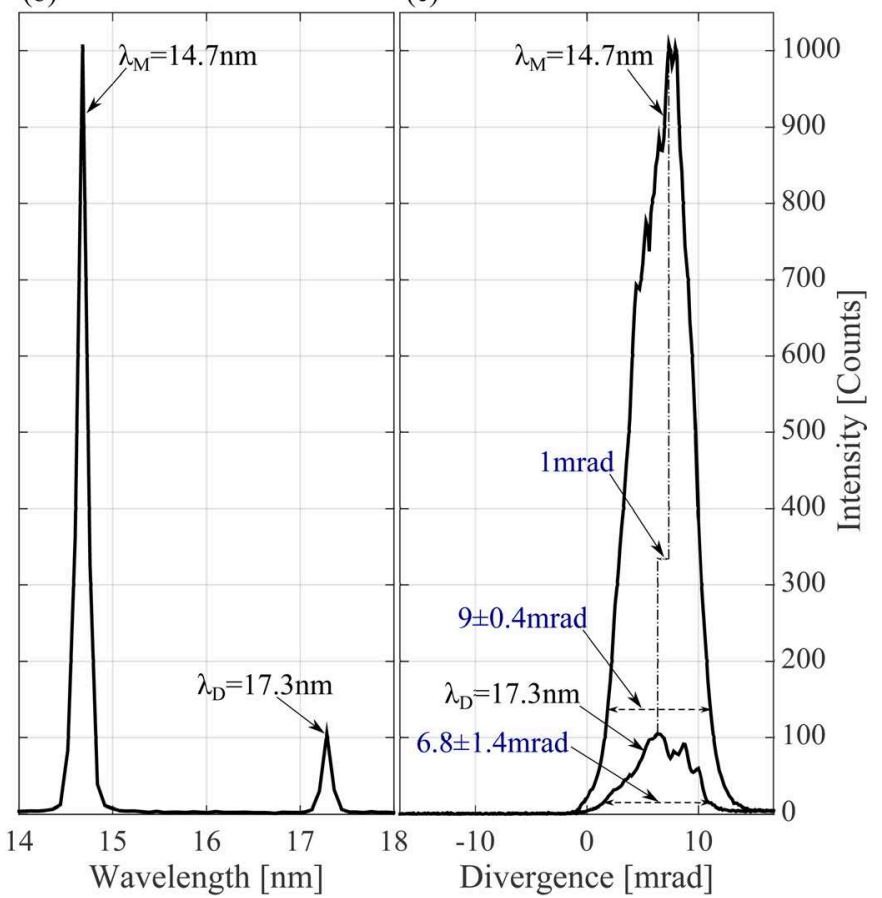

FIG. 6. (a) The experimentally measured mean intensity for lasing lines observed for Ni-like Pd at wavelengths $\lambda_{M}=14.7 \mathrm{~nm}$ and $\lambda_{D}=17.3 \mathrm{~nm}$. (b) Wavelength profile (vertical axis in (a)). (c) Divergence profile (horizontal axis in (a)). In subplots (a) and (c), the number 0 at the x-axis is the center of the image on the CCD-detector. 
TABLE II. Experimentally measured dual pulse characteristics (mean) of the XUV laser lines observed in the experiment for Pd.

\begin{tabular}{lccc}
\hline \hline $\begin{array}{l}\text { Wavelength } \\
(\mathrm{nm})\end{array}$ & $\begin{array}{c}\text { Divergence } \\
(\mathrm{mrad})\end{array}$ & $\begin{array}{c}\text { Pointing } \\
\text { stability (mrad) }\end{array}$ & $\begin{array}{c}\text { Relative bandwidth } \\
(\Delta \lambda / \lambda)\end{array}$ \\
\hline$\lambda_{M}=14.7$ & $9.0 \pm 0.4$ & 1.0 & $8 \times 10^{-3}$ \\
$\lambda_{D}=17.3$ & $6.8 \pm 1.4$ & 2.5 & $6 \times 10^{-3}$ \\
\hline \hline
\end{tabular}

Figs. 8(a) and 8(b) show the collisional excitation and de-excitation coefficients between the levels of a three-level laser model (monopole). Fig. 8(a) shows that for $T_{e} \gtrsim$ $200 \mathrm{eV}$ the dominant pumping is collisional from the coefficient $C_{02}$ (from the ground level to the upper level). It is noteworthy to mention that the hydro calculation shows that at lasing region population in the ground level is approximately 3.4 times higher than the population in the upper level. However, there is a gain because the population of the upper level is greater than the population of the lower level.

Fig. 8(a) shows that the monopole collisional excitation $\left(C_{02}\right)$ after $600 \mathrm{eV}$ begins to slightly decrease. Furthermore, it shows that $C_{02}$ has a saturation behavior with increasing temperature, i.e., $C_{02}$ increases $\approx 100$ times by increasing temperature from $50 \mathrm{eV}$ to $350 \mathrm{eV}$, meanwhile $C_{02}$ increases just $20 \%$ by increasing the temperature from $350 \mathrm{eV}$ to $600 \mathrm{eV}$.

Figs. 8(c) and 8(d) summarize the calculated collisional coefficients for population and de-population of the fourlevel laser model (dipole), as a function of the electron temperature. They show that with the increasing temperature the collisional de-excitation decreases meanwhile the collisional pumping increases, especially in the first hundreds of eV. Fig. 8(c) shows that for $T_{e} \gtrsim 400 \mathrm{eV}$ the dominant collisional-pumping is through the coefficient $C_{03}$ (from the ground level to the upper level).

Fig. 8(c) shows that dipole collisional excitation $\left(C_{03}\right)$ constantly increases by increasing temperature (up to $2000 \mathrm{eV}$ ). It shows that $C_{03}$ also has a saturation behavior with increasing temperature, i.e., $C_{03}$ increases $\approx 400$ times by increasing temperature from $50 \mathrm{eV}$ to $600 \mathrm{eV}$, meanwhile $C_{03}$ increases just $30 \%$ by increasing the temperature from $600 \mathrm{eV}$ to $1500 \mathrm{eV}$.

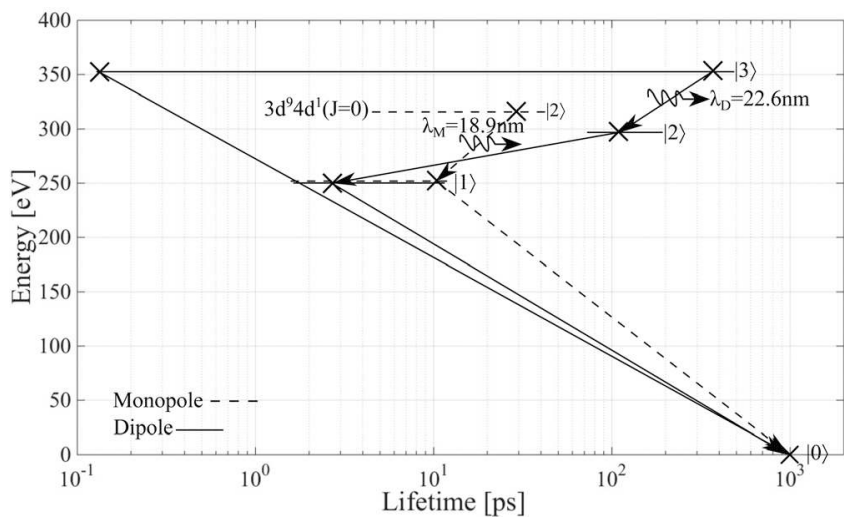

FIG. 7. Chrono-energy diagram for Ni-like Mo. The lifetime of each level due to the radiative decay is shown by a marker: $\times$. The three-level is for lasing due to monopole pumping (dashed lines). The four-level is for the lasing due to dipole pumping (solid lines).

\section{Time and space distribution of two-color gain}

Fig. 9(a) shows the gain as a function of time. The starting time corresponds to $1 \mathrm{ps}$ after arrival of the main-pulse (Fig. 9(a)). At the time of 2.4 ps, the peak of the main-pulse is deposited to the pre-plasma. The peaks of the gains produced by the two lines of $18.9 \mathrm{~nm}\left(E_{M}=65.6 \mathrm{eV}\right)$ and $22.6 \mathrm{~nm}$ $\left(E_{D}=54.9 \mathrm{eV}\right)$ happen at $2.7 \mathrm{ps}$ and $2.9 \mathrm{ps}$, respectively. Thus, the instant at which the maximum gain occurs have a time separation of $\approx 0.2 \mathrm{ps}$, i.e., the peak of the monopole XUV laser is $\approx 20 \%$ of pulse duration (pump pulse).

Fig. 9 shows that the gain at $\lambda_{M}=18.9 \mathrm{~nm}\left(E_{M}=65.6 \mathrm{eV}\right)$ is $\approx 4$ times higher than the gain at $\lambda_{D}=22.6 \mathrm{~nm}\left(E_{D}\right.$ $=54.9 \mathrm{eV})$. It shows a peak gain of $230 \mathrm{~cm}^{-1}$ at $\lambda_{M}=18.9 \mathrm{~nm}$ for the $4 d^{1}(J=0) \rightarrow 4 p^{1}(J=1)$ transition. It shows a peak gain of $55 \mathrm{~cm}^{-1}$ at $\lambda_{D}=22.6 \mathrm{~nm}$ with inclusion of the photopumping rate (Eq. (4)) for the $4 f^{1}(J=1) \rightarrow 4 d^{1}(J=1)$ transition. Meanwhile, the computation shows that by ignoring photo-pumping (Eq. (4)), no gain is obtained on the $4 \mathrm{f}^{1}(\mathrm{~J}=1)$ $\rightarrow 4 \mathrm{~d}^{1}(\mathrm{~J}=1)$ transition. Thus, the computational study shows that considering photo-pumping excitation improves the gain for the $4 \mathrm{f}^{1}(\mathrm{~J}=1) \rightarrow 4 \mathrm{~d}^{1}(\mathrm{~J}=1)$ transition.

Fig. 9(b) shows the gain as a function of space, i.e., height above the target. The peaks of the gain produced by two lines of $\lambda_{M}=18.9 \mathrm{~nm}\left(E_{M}=65.6 \mathrm{eV}\right)$ and $\lambda_{D}=22.6 \mathrm{~nm}$ $\left(E_{D}=54.9 \mathrm{eV}\right)$ are at heights $75.6 \mu \mathrm{m}$ and $70.4 \mu \mathrm{m}$, respectively. The active-medium thickness and gain lifetime are considered as the full width at half maximum of the gain, as shown in Figs. 9(a) and 9(b). Fig. 9(a) shows that the gain lifetime at $\lambda_{M}=18.9 \mathrm{~nm}\left(E_{M}=65.6 \mathrm{eV}\right)$ is a factor of $\approx 5$ larger than the gain lifetime at $\lambda_{D}=22.6 \mathrm{~nm}$. Fig. 9(b) shows that the gain thickness at $\lambda_{M}=18.9 \mathrm{~nm}\left(E_{M}=65.6 \mathrm{eV}\right)$ is a factor of $\approx 1.8$ larger than the gain thickness at $\lambda_{D}=22.6 \mathrm{~nm}$.

Fig. 9(a) shows that gain build-up time (required time to arrive maximum) due to collisional- and photo-pumping are 0.7 ps and 0.9 ps, respectively. Fig. 9(b) shows that the build-up length-scale of the gain (required length to arrive maximum) due to collisional- and photo-pumping is $11.5 \mu \mathrm{m}$ and $6.3 \mu \mathrm{m}$, respectively.

In Figs. 10(a)-10(c), the electron density, electron temperature, and photo-pumping rate versus height above the target at the times that maximize the gains due to the collisional- and photo-pumping are shown, respectively.

Fig. 9(b) (see x-axis) confirms that the gain-area at wavelength $\lambda_{D}$ overlaps quite well the gain-area at wavelength $\lambda_{M}$. It shows that the maximum electron density within the gain region for both wavelengths is the same. Then, considering the refraction angle in Eq. (15) yields

$$
\frac{\phi_{\mathrm{r}-18.9 \mathrm{~nm}}}{\phi_{\mathrm{r}-22.6 \mathrm{~nm}}}=\sqrt{\frac{n_{\mathrm{c}-22.6 \mathrm{~nm}}}{n_{\mathrm{c}-18.9 \mathrm{~nm}}}}=1.2,
$$

which shows that the refraction angle at $\lambda_{M}$ is $20 \%$ times larger than the refraction angle at $\lambda_{D}$. This is in agreement with the experimental results (see Sec. IV A 1). In Sec. IV A 1 , it is shown that the experimentally measured divergence of $\lambda_{M}=18.9 \mathrm{~nm}$ is $\approx 1.2$ times larger than the one of $\lambda_{D}=22.6 \mathrm{~nm}$.

Fig. 10(a) shows that lasing of both XUV lines (monopole and dipole) occurs directly and very efficiently in the 

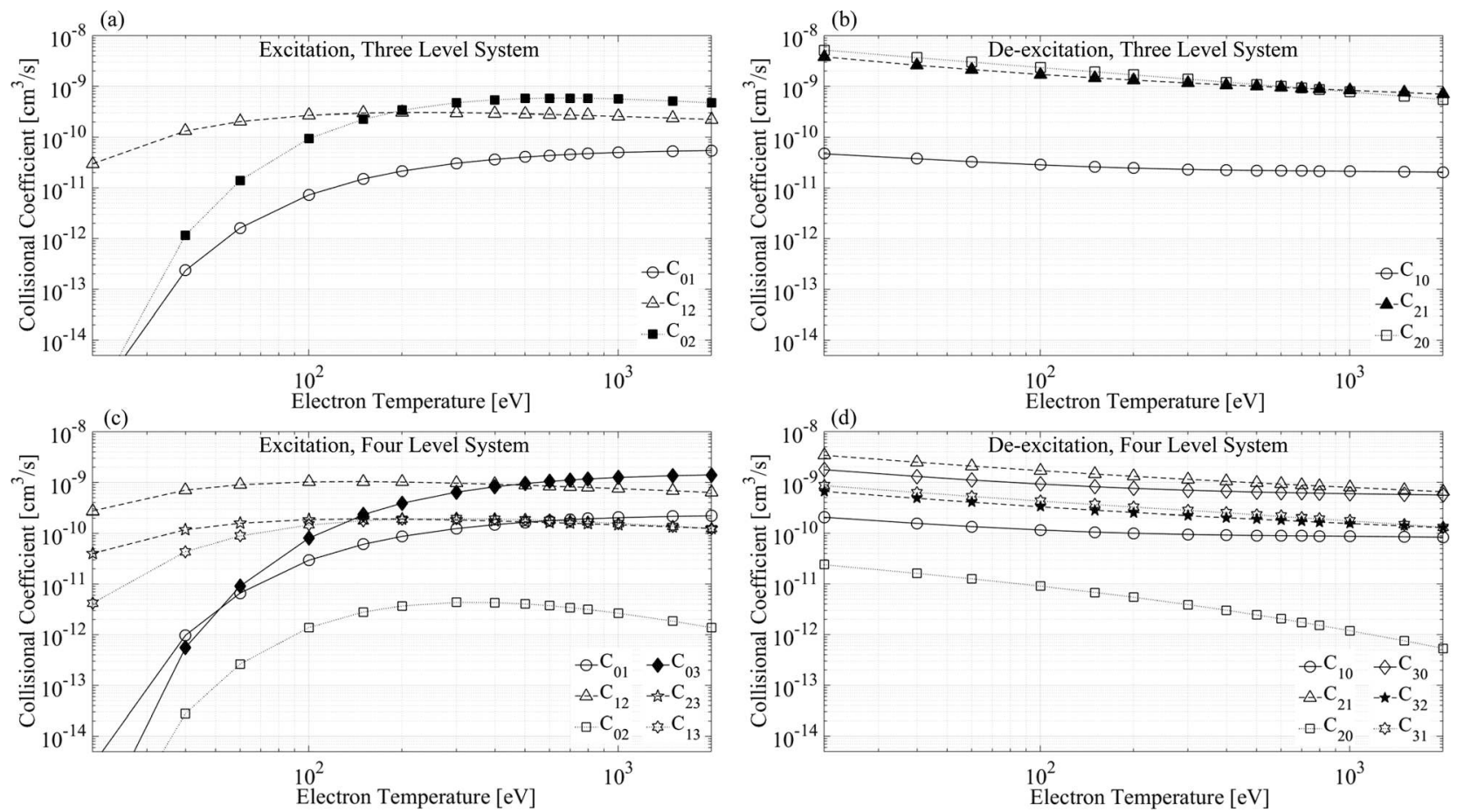

FIG. 8. Calculated collisional (a) excitation and (b) de-exciation coefficients for a three-level system as a function of the electron temperature for monopole lasing in Ni-like Mo. Calculated collisional (c) excitation and (d) de-exciation coefficients for four-level system as a function of the electron temperature for dipole lasing in Ni-like ions.

region beyond the turning point to heat up a plasma in order to achieve the optimum temperature required to produce strong pumping to the upper laser level, allowing the lasing process to begin. Considering $\theta=40^{\circ}$ (see Fig. 3), the electron density at the turning point of the pump pulse is

$$
n_{t e}=n_{c} \cos ^{2} \theta \approx 5.9 \cdot 10^{20} \mathrm{~cm}^{-3}
$$

where the critical density for the pump pulse is $n_{c} \simeq 10^{21} \mathrm{~cm}^{-3}$.

Fig. 10(c) shows that at the point maximum gain the photo-pumping rate is also at its maximum value, which is $R_{03}^{p} \approx 1.5 \times 10^{13} \mathrm{~s}^{-1}\left(\tau_{03}^{p}=1 / R_{03}^{p}=67 \mathrm{fs}\right)$.

Table III summarizes the gain lifetime, gain thickness, the electron density, and the electron temperature maximizing the gain, and the phase velocity $\left(v_{p}\right)$ for doublecolor in Ni-like Mo plasma.

As it is shown in Table III, the electron density and electron temperature corresponding to the maximum gain at wavelength $\lambda_{D}$ are 1.7 and 1.2 times higher than the electron density and electron temperature which maximize the gain at wavelength $\lambda_{M}$.

By considering the electron densities in Table III and using Eq. (15), divergences at the points of maximum gain for dipole and monopole transitions are obtained, respectively, as $9.8 \mathrm{mrad}$ and $6.2 \mathrm{mrad}$, a difference of $3.6 \mathrm{mrad}$. It is in agreement with the experimental result. As it is shown in Fig. 5(c), the mean peak intensity of two-color occurs with $3.2 \mathrm{mrad}$ difference.
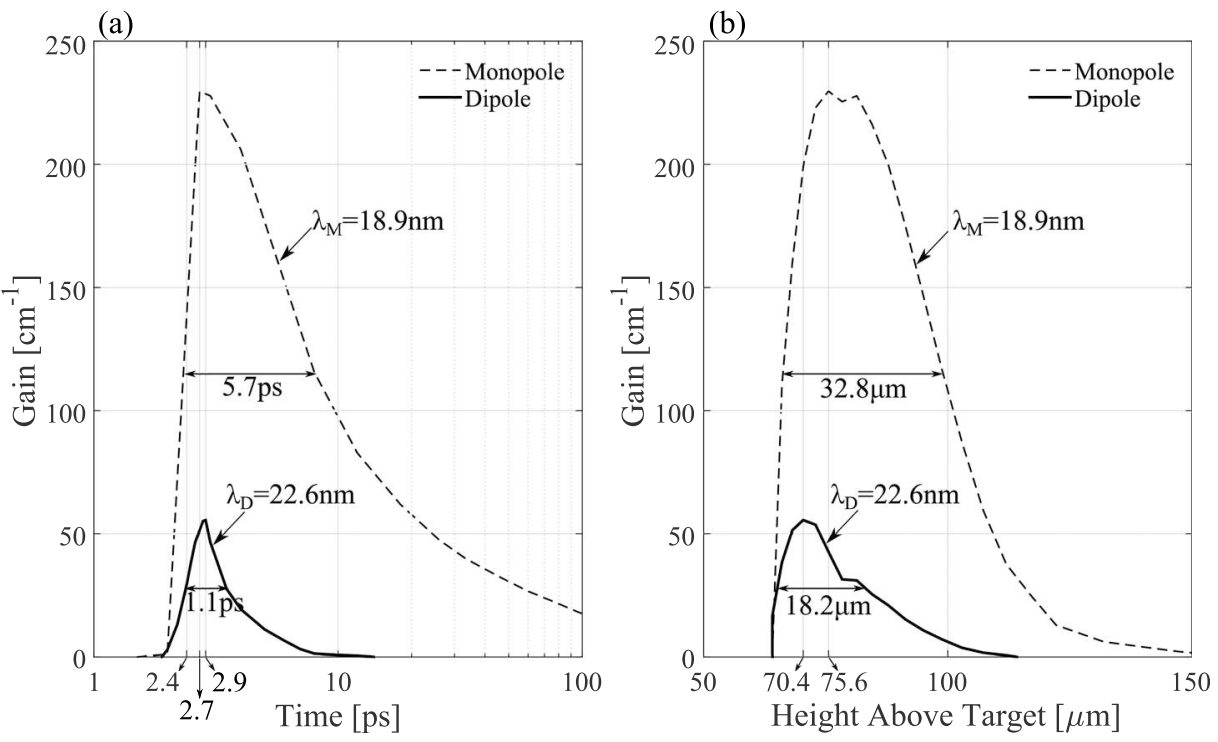

FIG. 9. (a) Temporally resolved and (b) spatially resolved calculated gain for the transitions $4 \mathrm{~d}^{1}(\mathrm{~J}=0) \rightarrow 4 \mathrm{p}^{1}$ $(\mathrm{J}=1), \quad \lambda_{M}=18.9 \mathrm{~nm}$, and $4 \mathrm{f}^{1}(\mathrm{~J}=1)$ $\rightarrow 4 \mathrm{~d}^{1}(\mathrm{~J}=1), \lambda_{D}=22.6 \mathrm{~nm}$. (a) The starting time in $\mathrm{x}$-axis is from $1 \mathrm{ps}$, which corresponds to the $1 \mathrm{ps}$ after arrival of main-pulse. (a) Time $2.4 \mathrm{ps}$ corresponds to the arrival time of the peak of the main-pulse. 

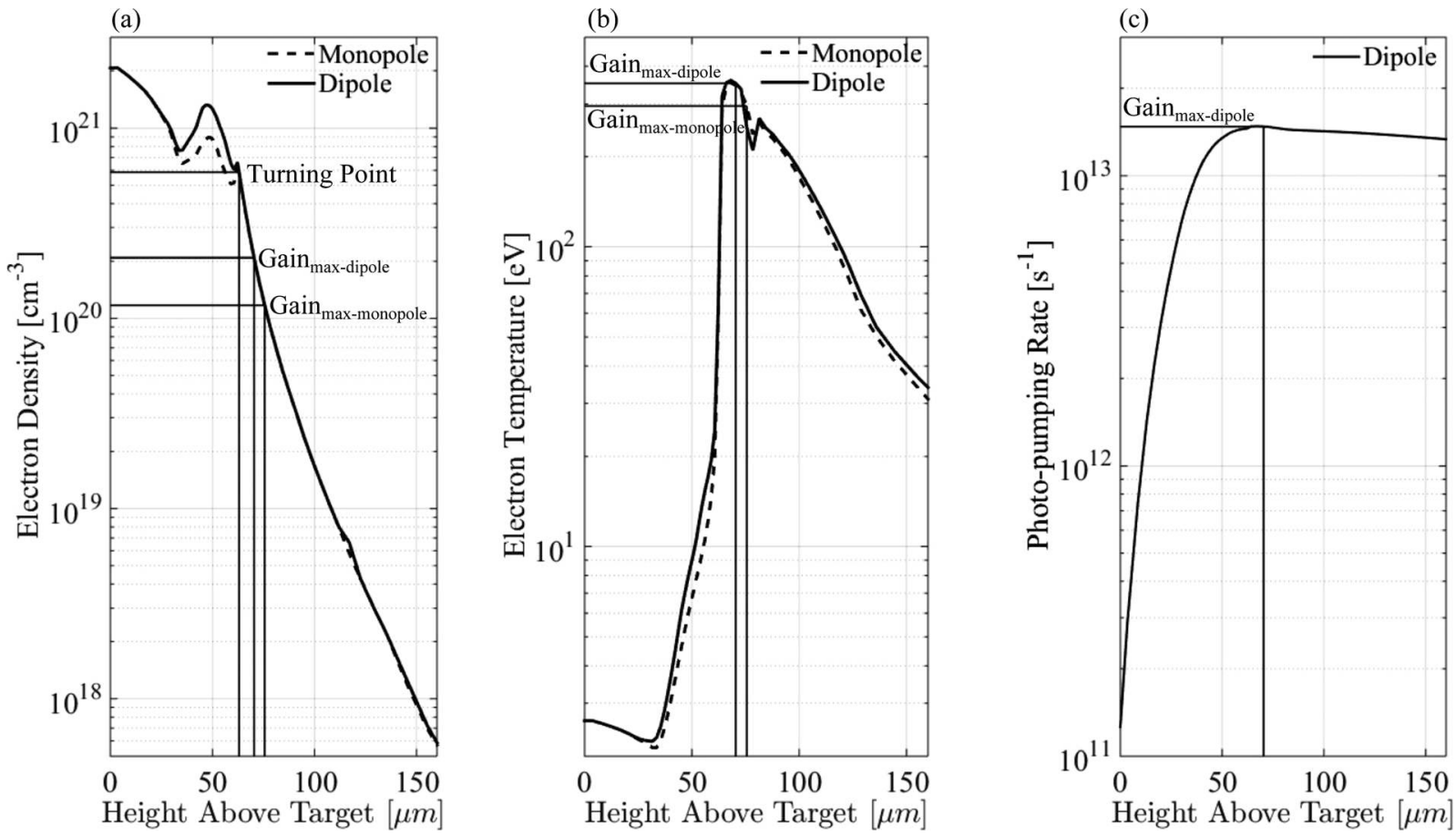

FIG. 10. (a) Electron density and (b) electron temperature of lasing Mo at the time corresponding to maximum gain for the monopole $\left(\lambda_{M}=18.9 \mathrm{~nm}\right)$ and dipole $\left(\lambda_{D}=22.6 \mathrm{~nm}\right)$ transition are shown, which are calculated with the hydrodynamics code. (c) By calculating the radiation temperature with the hydrodynamics code, photo-pumping rate (Eq. (4)) at the time corresponding to maximum gain for the dipole transition is obtained. The height above the target is in the direction normal to the surface of target. The peaks of the gain (Gain max $_{\text {) }}$ produced by two transitions are at heights $70.4 \mu \mathrm{m}$ (dipole) and $75.6 \mu \mathrm{m}$ (monopole) above the target.

Table III shows the phase velocity at these two points. The phase velocity of XUV laser in the plasma is calculated by $v_{p}=c / n$, where $n$ is the refractive index of plasma. The critical densities $\left(n_{c}\right)$ at wavelengths $\lambda_{D}=22.6 \mathrm{~nm}$ and $\lambda_{M}=18.9 \mathrm{~nm}$ are $2.2 \times 10^{24} \mathrm{~cm}^{-3}$ and $3.1 \times 10^{24} \mathrm{~cm}^{-3}$, respectively. It shows that the phase velocity at the point of maximum gain for dual color lasing has a difference of $\approx 3.3$ $\times 10^{-5} \mathrm{c}$.

Taking into account the electron density profile that maximizes gain (see Fig. 10(a)), the monopole and dipole XUV laser trajectories along the plasma gain medium can be calculated and is shown in Fig. 11(a). Fig. 11(a) shows that the monopole XUV trajectory is longer, which will maximize the amplification length.

The gain-length product along the target can be obtained by the combination of a ray tracing calculation (see Fig. 11(a)) and gain calculation (see Fig. 9).

Fig. 11(b) shows the small signal gain-length product for monopole- and dipole-pumping, respectively. In fact, by knowing the gain above the target and using the ray trajectory equation, the gain-length product is calculated along the target. In Fig. 11(b), the gain-length product is shown.
Fig. 11 shows $\left(g_{0} L\right)_{\text {monopole }} /\left(g_{0} L\right)_{\text {dipole }} \approx 8$ (Eq. (11)). The saturated intensity (Eq. (9)) of the monopole laser line is three times larger than saturated intensity of dipole one. Thus, considering Eq. (10) shows that the total intensity of monopole pulse is $\approx 24$ times larger than the total intensity of dipole pulse. In Fig. 5, the integration of the experimentally measured intensity profile of monopole and dipole lines shows that the total intensity of the monopole pumping is $\approx 25$ times larger than dipole one. Then, a good agreement between the computational and experimental results is proved, where $\left(I_{\text {tot }_{M}} / I_{\text {tot }_{D}}\right)_{\text {computation }} \approx 24$ and $\left(I_{\text {tot }_{M}} / I_{\text {tot }_{D}}\right)_{\text {experiment }} \approx 25$.

\section{Discrete tunability of the two-color XUV laser}

The two XUV lines for Ni-like Mo and Pd were measured experimentally here. Complete computational analysis of Mo target has been presented. Here, it is shown that by changing the target material the two-color XUV laser can be made tunable over the entire soft X-ray domain. Table IV shows the photo-pumping wavelengths for some other $\mathrm{Ni}$ like atoms calculated by atomic calculations (FAC) and experiment. Since calculations are approximations, the

TABLE III. Calculated gain lifetime at the $4 \mathrm{~d}^{1}(\mathrm{~J}=0) \rightarrow 4 \mathrm{p}^{1}(\mathrm{~J}=1)$ (monopole) and $4 \mathrm{f}^{1}(\mathrm{~J}=1) \rightarrow 4 \mathrm{~d}^{1}(\mathrm{~J}=1)$ (dipole) two-color XUV for the Ni-like Mo laser. Calculated electron temperature $\left(T_{e}\right)$ and electron density $\left(n_{e}\right)$ maximizing gain at each color are presented. Phase velocity $\left(v_{p}\right)$ and refraction angle $\left(\phi_{r}\right)$ at the points corresponding to the maximum gain for dipole and monopole transitions are presented.

\begin{tabular}{lcccccrr}
\hline \hline$\lambda_{x}(\mathrm{~nm})$ & Gain peak $\left(\mathrm{cm}^{-1}\right)$ & Gain lifetime $(\mathrm{ps})$ & Gain thickness $(\mu \mathrm{m})$ & $n_{e}\left(\mathrm{~cm}^{-3}\right)$ & $T_{e}(\mathrm{eV})$ & $\phi_{r}(\mathrm{mrad})$ & $v_{p}$ \\
\hline$\lambda_{M}=18.9$ & 230 & 5.7 & 32.8 & $1.2 \times 10^{20}$ & 295 & 6.2 & $\left(1+1.9 \times 10^{-5}\right) c$ \\
$\lambda_{D}=22.6$ & 55 & 1.1 & 18.2 & $2.1 \times 10^{20}$ & 350 & 9.8 & $\left(1+5.2 \times 10^{-5}\right) c$ \\
\hline \hline
\end{tabular}



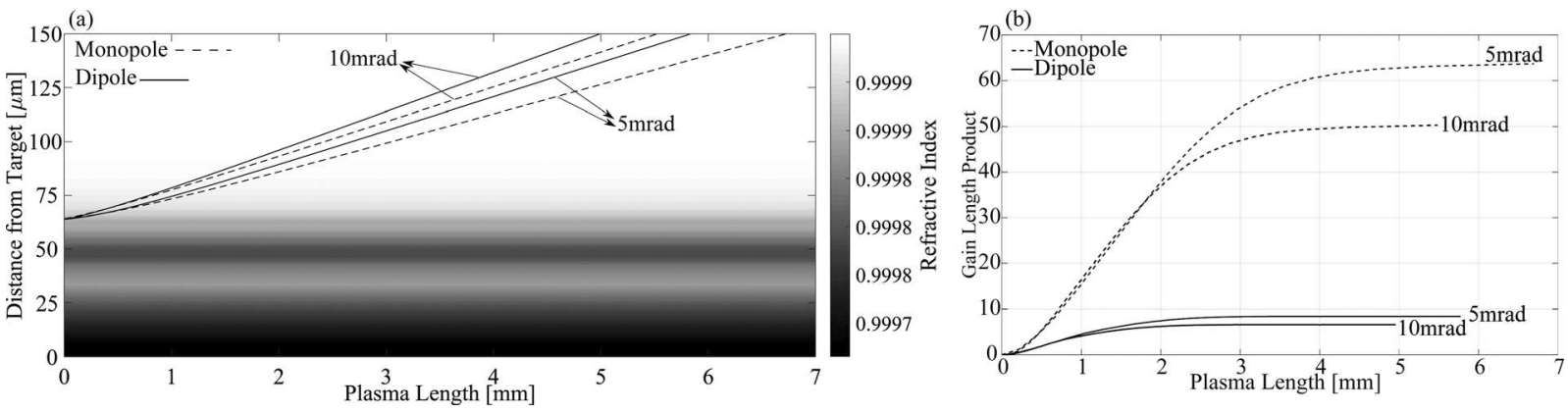

FIG. 11. (a) Short-wavelength laser trajectory along the plasma gain medium. The monopole X-ray pulse remains within the plasma gain region for longer. (b) Gain-length product of monopole and dipole lines (Eq. (11)).

TABLE IV. Calculated wavelengths due to photo-pumping in Ni-like ions. The experimentally measured wavelengths by other literatures ${ }^{15,16}$ are presented. The experimentally measured wavelengths for Mo and Pd in this work are presented. $\lambda_{\text {D-FAC }}$ gives a value calculated by the FAC code. $\lambda_{D}$ is a value estimated by considering a correction factor, which is obtained by comparison of experimentally and computationally calculated wavelengths.

\begin{tabular}{lccc}
\hline \hline Ni-like ion & $\begin{array}{c}\lambda_{\text {D-FAC }} \\
(\mathrm{nm})\end{array}$ & $\begin{array}{c}\lambda_{D}(\mathrm{~nm}) \\
(\text { Correction factor })\end{array}$ & $\begin{array}{c}\lambda_{D}(\mathrm{~nm}) \\
(\text { Experiment })\end{array}$ \\
\hline $\mathrm{Y}^{+11}(Z=39)$ & 28.8 & 30.4 & $\ldots$ \\
$\mathrm{Zr}^{+12}(Z=40)$ & 26.0 & 27.1 & $27.1^{16}$ \\
$\mathrm{Nb}^{+13}(Z=41)$ & 23.7 & 24.6 & $24.6^{15}$ \\
$\mathrm{Mo}^{+14}(Z=42)$ & 21.8 & 22.6 & 22.6 (this work) \\
$\operatorname{Pd}^{+18}(Z=46)$ & 16.4 & 17.3 & 17.3 (this work) \\
$\mathrm{Ag}^{+19}(Z=47)$ & 15.5 & 16.3 & $\ldots$ \\
$\mathrm{Cd}^{+20}(Z=48)$ & 14.7 & 15.5 & $\ldots$ \\
$\mathrm{In}^{+21}(Z=49)$ & 13.9 & 14.7 & $\ldots$ \\
$\operatorname{Sn}^{+22}(Z=50)$ & 13.2 & 14.0 & $\ldots$ \\
$\mathrm{Te}^{+24}(Z=52)$ & 12.1 & 12.7 & $\ldots$ \\
$\operatorname{Sm}^{+34}(Z=62)$ & 8.4 & 8.8 & $\ldots$ \\
\hline \hline
\end{tabular}

correction factor is considered on the computationally calculated wavelengths (Table IV). Photo-pumping wavelengths with the correction factor can be predicted based on the comparison between the wavelengths calculated by FAC and the wavelengths obtained experimentally. Table IV shows that with increasing the atomic number the estimated wavelengths are closer to those calculated by atomic calculations (FAC) due to fact that the FAC is a code based on the $j-j$ coupling which better described for high- $Z$ atoms or heavily ionized atoms.

\section{CONCLUSIONS}

Plasma-driven XUV lasers offer the possibility of multiple transitions in a target material to generate a two-color XUV laser in a table-top setup. Indeed, such a mode of operation is not simple at all in accelerator facilities for X-ray laser sources, such as Free electron lasers. In this work, experimental and computational results on the two-color XUV lasing using a Ni-like plasma as a gain medium were shown. We highlighted the atomic physics of the collisionally pumped laser (monopole pumping) versus the photopumped laser (dipole pumping).

Both computational and experimental results showed that the divergence of the monopole excited XUV pulse is
$20 \%$ larger than the divergence for the pulse corresponding to the dipole transition. The mean peak intensity of the two colors (monopole and dipole) is separated on the far field by $3.2 \mathrm{mrad}$. Computational results show that the refraction angle at the points corresponding to the maximum gain for dipole and monopole transitions are, respectively, as 9.8 $\mathrm{mrad}$ and $6.2 \mathrm{mrad}$, i.e., a significant difference in light propagation across the inhomogeneous plasma gain-medium. The plasma that maximizes the dipole transition rate is hotter and denser than the plasma that optimizes the monopole transition rate. The electron density and electron temperature maximizing the gain at $\lambda_{D}$ are a factor of 1.7 and 1.2 higher than the electron density and electron temperature maximizing the gain at $\lambda_{M}$. It is demonstrated computationally that the peaks of the gain for both $\lambda_{M}$ and $\lambda_{D}$ form after the turning point at heights of $75.6 \mu \mathrm{m}$ and $70.4 \mu \mathrm{m}$ above the target.

This computational study showed that the peak of the gain of two colors (monopole and dipole) occurs at the time difference of $\Delta t \approx 0.2 \mathrm{ps}$. The gain of monopole is a factor of 4 higher than the gain for the dipole. The gain lifetime at $\lambda_{M}$ is a factor of $\approx 5$ larger than the gain lifetime at $\lambda_{D}$. The gain thickness at $\lambda_{M}$ is a factor of $\approx 1.8$ larger than the gain thickness at $\lambda_{D}$. It is shown that the gain build-up time due to the collisional- and photo-pumping is $0.7 \mathrm{ps}$ and $0.9 \mathrm{ps}$, respectively. Our results indicate that the build-up length-scale of the gain due to collisional- and photo-pumping is $11.5 \mu \mathrm{m}$ and $6.3 \mu \mathrm{m}$, respectively. It is demonstrated that ignoring photo-pumping, no gain is obtained on the $3 \mathrm{~d}^{9} 4 \mathrm{f}^{1}(\mathrm{~J}=1) \rightarrow$ $3 \mathrm{~d}^{9} 4 \mathrm{~d}^{1}(\mathrm{~J}=1)$ transition. We have extrapolated our study to a larger set of target material, in order to highlight the potential for tunability.

\section{ACKNOWLEDGMENTS}

The present work was supported by the Swiss National Science Foundation under Grant No. PP00P2-133564/1.

The authors wish to acknowledge the comments offered by Dr. Jakub Szlachetko (Paul Scherrer Institut) and Dr. Claudio Cirelli (Empa).

${ }^{1}$ A. A. Lutman, R. Coffee, Y. Ding, Z. Huang, J. Krzywinski, T. Maxwell, M. Messerschmidt, and H. D. Nuhn, Phys. Rev. Lett. 110, 134801 (2013).

${ }^{2}$ T. Hara, Y. Inubushi, T. Katayama, T. Sato, H. Tanaka, T. Tanaka, T. Togashi, K. Togawa, K. Tono, M. Yabashi, and T. Ishikawa, Nat Commun. 4, 2919 (2013).

${ }^{3}$ M. Dantus, R. M. Bowman, and A. H. Zewail, Nature 343, 737 (1990).

${ }^{4}$ S. Benkovic and S. Hammes-Schiffer, Science 301(5637), 1196 (2003). 
${ }^{5}$ R. M. van der Veen, J. J. Kas, C. J. Milne, V. T. Pham, A. El Nahhas, F. A. Lima, D. A. Vithanage, J. J. Rehr, R. Abela, and M. Chergui, Phys. Chem. Chem. Phys. 12(21), 5551 (2010).

${ }^{6}$ J. Szlachetko, M. Nachtegaal, J. Sá, J.-Cl. Dousse, J. Hoszowska, E. Kleymenov, M. Janousch, O. V. Safonova, C. König, and J. A. van Bokhoven, Chem. Commun. 48, 10898 (2012).

${ }^{7}$ J. Szlachetko, C. J. Milne, J. Hoszowska, J.-Cl. Dousse, W. B. lachucki, J. Sá, Y. Kayser, M. Messerschmidt, R. Abela, S. Boutet, C. David, G. Williams, M. Pajek, D. B. Patterson, G. Smolentsev, J. A. Van Bokhoven, and M. Nachtegaal, Struct. Dyn. 1, 021101 (2014).

${ }^{8}$ A. Thompson, D. Attwood, E. Gullikson, M. Howells, K-J. Kim, J. Kirz, J. Kortright, I. Lindau, P. Pianetta, A. Robinson, J. Scofield, J. Underwood, D. Vaughan, G. Williams, and H. Winick, X-Ray Data Booklet (Lawrence Berkeley National Laboratory, Berkeley, 2009).

${ }^{9}$ A. Marinelli and M. Park, in Proceedings of IPAC, THPPA01, Dresden, Germany (2014).

${ }^{10}$ A. Marinelli, A. A. Lutman, J. Wu, Y. Ding, J. Krzywinski, H. D. Nuhn, Y. Feng, R. N. Coffee, and C. Pellegrini, Phys. Rev. Lett. 111, 134801 (2013).

${ }^{11}$ A. Marinelli, D. Ratner, A. A. Lutman, J. Turner, J. Welch, F. J. Decker, H. Loos, C. Behrens, S. Gilevich, A. A. Miahnahri, S. Vetter, T. J. Maxwell, Y. Ding, R. Coffee, S. Wakatsuki, and Z. Huang, Nat. Commun. 6, 6369 (2015).

${ }^{12}$ Z. Zhang, Y. Ding, A. Marinelli, and Z. Huang, Phys. Rev. Spec. Top. Accel. Beams 18, 030702 (2015).

${ }^{13}$ D. Bleiner, "Short-wavelength imaging and spectroscopy sources," Proc. SPIE 8678, 867806 (2012).

${ }^{14}$ D. Bleiner, "Coherent short-wavelength plasma radiation for lab-scale nano-inspection tools," in Proceedings of the ICXRL 2012 (2012), Chap. 7, Vol. 147, p. 39.

${ }^{15}$ J. Nilsen, J. Dunn, A. L. Osterheld, and Y. Li, Phys. Rev. A 60, R2677 (1999).

${ }^{16}$ Y. Li, J. Nilsen, J. Dunn, A. L. Osterheld, A. Ryabtsev, and S. Churilov, Phys. Rev. A 58(4), R2668 (1998).

${ }^{17}$ J. Nilsen, J. Opt. Soc. Am. B 14(6), 1511 (1997).

${ }^{18}$ D. Bleiner and T. Feurer, Appl. Opt. 51(36), 8848 (2012).

${ }^{19}$ D. Bleiner, J. E. Balmer, and F. Staub, Appl. Opt. 50, 6689 (2011)

${ }^{20}$ D. Bleiner, Y. Arbelo-Pena, L. Masoudnia, and M. Ruiz-Lopez, Phys. Scr., T162, 014050 (2014).

${ }^{21}$ T. Kita, T. Harada, N. Nakano, and H. Kuroda, Appl. Opt. 22, 512 (1983).

${ }^{22} \mathrm{~J}$. T. Larsen, HYADES-A Plasma Hydrodynamics Code for Dense Plasma Studies (Cascade Applied Sciences Inc., 1990).

${ }^{23}$ J. T. Larsen and S. M. Lane, J. Quant. Spectrosc. Radiat. Transfer 51, 179 (1994).

${ }^{24}$ See http://www.clf.stfc.ac.uk/CLF/resources/PDF/FullArticles/47.pdf for "R. H. H. Scott, N. Booth, S. J. Hawkes, D. Symes, H. W. Doyle, S. I. Olsson-Robbie, H. F. Lowe, C. Price, D. Bigourd, S. Patankar, and K. Mecseki, Modelling radiative-shocks created by laser-cluster interactions" (accessed 4 April, 2016).
${ }^{25}$ R. A. Ganeev, J. Phys. B 40(22), R213 (2007).

${ }^{26}$ J. P. Holloway, D. Bingham, C.-C. Chou, F. Doss, R. P. Drake, B. Fryxell, M. Grosskopf, B. van der Holst, B. K. Mallick, R. McClarren, A. Mukherjee, V. Nair, K. G. Powell, D. Ryu, I. Sokolov, G. Toth, and Z. Zhang, Reliab. Eng. Syst. Saf. 96(9), 1184 (2011).

${ }^{27}$ A. M. Rubenchik, M. D. Feit, M. D. Perry, and J. T. Larsen, Appl. Surf. Sci. 127-129, 193 (1998).

${ }^{28}$ L. Masoudnia and D. Bleiner, Laser Phys. 23, 056003 (2013).

${ }^{29}$ L. Masoudnia and D. Bleiner, Nucl. Instrum. Methods Phys. Res., B 323, 59 (2014).

${ }^{30}$ M. F. Gu, "The flexible atomic code (FAC)," Can. J. Phys. 86, 675 (2008).

${ }^{31}$ M. Bitter, M. F. Gu, L. A. Vainshtein, P. Beiersdorfer, G. Bertschinger, O. Marchuk, R. Bell, B. LeBlanc, K. W. Hill, D. Johnson et al., Phys. Rev. Lett. 91, 265001 (2003).

${ }^{32}$ X. L. Wang, C. Z. Dong, and M. G. Su, Nucl. Instrum. Methods Phys. Res., B 280, 93 (2012).

${ }^{33}$ M. J. May, P. Beiersdorfer, N. Jordan, J. H. Scofield, K. J. Reed, S. B. Hansen, K. B. Fournier, M. F. Gu, G. V. Brown, F. S. Porter et al., Nucl. Instrum. Methods Phys. Res., B 235, 231 (2005).

${ }^{34}$ K. M. Aggarwal and F. P. Keenan, At. Data Nucl. Data Tables 99, 156 (2013).

${ }^{35}$ J. Nilsen, Phys. Rev. A 53(6), 4539 (1996).

${ }^{36}$ P. L. Hagelstein, Plasma Phys. 25, 1345 (1983).

${ }^{37}$ R. C. Elton, X-Ray Lasers (Academic Press Inc., London, 1990).

${ }^{38}$ J. J. Olivero and R. L. Longbothum, J. Quant. Spectrosc. Radiat. Transfer 17, 233 (1977).

${ }^{39}$ H. R. Griem, Principle of Plasma Spectroscopy (Cambridge University Press, New York, 1997).

${ }^{40}$ R. A. London, Phys. Fluids 31, 184 (1988).

${ }^{41}$ S. De. Silvestri, M. Nisoli, G. Sansone, S. Stagira, and C. Vozzi, Strong Field Laser Physics, Springer Series in Optical Sciences Vol. 134, edited by T. Brabec (Springer-Verlag, New York, 2009).

${ }^{42}$ P. W. Milonni and J. H. Eberly, Laser Physics (Wiley, New Jersey, 2010).

${ }^{43}$ F. Trager, Springer Handbook of Lasers and Optics (Springer, 2007).

${ }^{44}$ R. Keenan, J. Dunn, V. N. Shlyaptsev, R. F. Smith, P. K. Patel, and D. F. Price, Proc. SPIE 5197, 213 (2003).

${ }^{45}$ J. Dunn, R. Keenan, P. K. Patel, D. F. Price, R. F. Smith, and V. N. Shlyaptsev, in Proceeding on X-Ray Lasers, edited J. Zhang (Taylor and Francis, London, 2004).

${ }^{46}$ M. Ruiz-Lopez and D. Bleiner, Appl. Phys. B 115(3), 311 (2014).

${ }^{47}$ G. V. Marr, Handbook on Synchrotron Radiation (North-Holland, 1987), Vol. 2.

${ }^{48}$ R. Follath, F. Senf, and W. Gudat, J. Synchrotron Rad. 5, 769 (1998).

${ }^{49}$ B. Y. Man, Q. L. Dong, A. H. Liu et al., J. Opt. A: Pure Appl. Opt. 6, 17 (2004).

${ }^{50}$ G. J. Pert, Phys. Rev. A 73, 033809 (2006). 